\title{
A Navigation System for Augmenting Laparoscopic Ultrasound
}

\author{
James Ellsmere ${ }^{1,2}$, Jeffrey Stoll ${ }^{1,3}$, David Rattner ${ }^{1,2}$, David Brooks ${ }^{2}$, Robert Kane ${ }^{2}$, \\ William Wells ${ }^{2}$, Ron Kikinis ${ }^{1,2}$, and Kirby Vosburgh ${ }^{1}$ \\ ${ }^{1}$ Center for Integration of Medicine and Innovative Technology, Cambridge MA \\ \{drattner, dbrooks, kvosburgh\}@partners.org \\ ${ }^{2}$ Departments of Surgery and Radiology, Harvard Medical School, Boston MA \\ \{jellsmere, sw, kikinis\}@bwh.harvard.edu, \\ rkane@caregroup.harvard.edu \\ ${ }^{3}$ Department of Mechanical Engineering, Boston University, Boston MA \\ jstoll@bu.edu
}

\begin{abstract}
Establishing image context is the major difficulty of performing laparoscopic ultrasound. The standard techniques used by transabdominal ultrasonographers to understand image orientation are difficult to apply with laparoscopic instruments. In this paper, we describe a navigation system that displays the position and orientation of laparoscopic ultrasound images to the operating surgeon in real time. The display technique we developed for showing the orientation information uses a 3D model of the aorta as the main visual reference. This technique is helpful because it provides surgeons with important spatial cues, which we show improves their ability to interpret the laparoscopic ultrasound.
\end{abstract}

\section{Introduction}

Laparoscopy revolutionized the practice of abdominal surgery. Many open operations were replaced with less morbid, minimally invasive procedures. Today, surgical patients can often return home the same day and return to work in days, as compared to weeks with conventional surgery. This led surgeons to investigate combining other imaging modalities, such as ultrasound (US), with laparoscopy to expand the range of minimally invasive procedures. Over the last five years, several important applications for laparoscopic US have emerged, including: improved staging of hepatic and pancreatic malignancies and assessing the common bile duct for stones. Despite these advances, surgeons have been slow to adopt laparoscopic US. The major obstacle facing surgeons learning to use laparoscopic US is understanding how the US images are oriented relative to the patient [1-4].

The basis of this problem is that the orientation techniques used by transabdominal ultrasonographers are difficult to apply with laparoscopic instruments. Both novice and experienced ultrasongraphers learning to use laparoscopic US struggle to understand the position and orientation of the B-scan plane. With transabdominal US, orienting an image is often accomplished by using standardized imaging planes (e.g., 
longitudinal, axial). When standardized planes are not possible because of limited acoustic windows, the technique for orienting an oblique image is to first image a landmark in a known plane and then move the transducer to region of interest [4]. With this technique, ultrasongraphers can be confident they know where they are looking because they understand where they started and how they moved the transducer. With laparoscopic US, however, both the intraoperative acoustic windows and the allowable movement of the probe are highly constrained. As a result, most images are from oblique planes. In addition, the movements for controlling laparoscopic probes are more complex than for transabdominal probes. Since the laparoscopic access port acts as a fixed fulcrum, some hand movements are inverted, some are exaggerated, and some remain the same. These mechanical constraints make the standard US orientation techniques very difficult to apply.

Other groups have attempted to address the laparoscopic US visualization problem using intraoperative 3D US [3]. Harms' group used a magnetically tracked laparoscopic US to generate 3D US volumes of intra-abdominal pathology. They concluded that their visualization technique was helpful but that they required image segmentation in the operating room. Routine volume rendering was not helpful because of low tissue contrast [5]. Since robust automated 3D US segmentation techniques are not available and manual segmenting is not feasible in the operating room, it seems 3D US alone is not adequate to overcome this problem.

The technique we developed is to provide orientation by rendering the laparoscopic US plane relative to an aortagram. First, we generate 3D models of the laparoscopic transducer, the B-scan plane, the aorta and the ribs. Then we register these models with the real objects in the operating room. Lastly, we track the laparoscopic transducer and render the position and orientation of the US plane as it changes relative to the aorta and ribs. Figure 1 shows two examples of the display in use. This technique is helpful because it provides physicians with important spatial cues that ultimately improve their ability to interpret the US images [6].

The key component to this visualization technique is the model of the aorta and its major branches. The aorta makes an excellent reference because it runs throughout the entire volume of interest and it has characteristic 3D shape that surgeons quickly identify [7]. As well, it is relatively fixed and non-deformable so it can be registered using rigid techniques [8]. Unlike most intraabdominal structures (e.g., stomach, bowel, liver and spleen), the aorta is held to the spine by connective tissue so its physiological motion limited to a few millimeters. The aorta is resistant to deformation because of high intraluminal pressure.

We tested the spatial accuracy of the system by generating 3D US volumes of a radiological phantom and comparing the volumes to CT scans of the same phantom. We tested the feasibility and utility of the system in an animal laboratory by having four physicians perform a laparoscopic US exam on a pig and assessing whether the system improved their ability to identify key landmarks. We further tested the utility of the system by recording a laparoscopic US exam and performing off line testing. To record the exam, we simultaneously digitized the video from the laparoscopic camera, the laparoscopic US and the navigation system. We had 20 surgeons review the digital video recordings and assessed whether their ability to identify key landmarks was effected by whether they were provided with the orientation display. Our results are presented. 

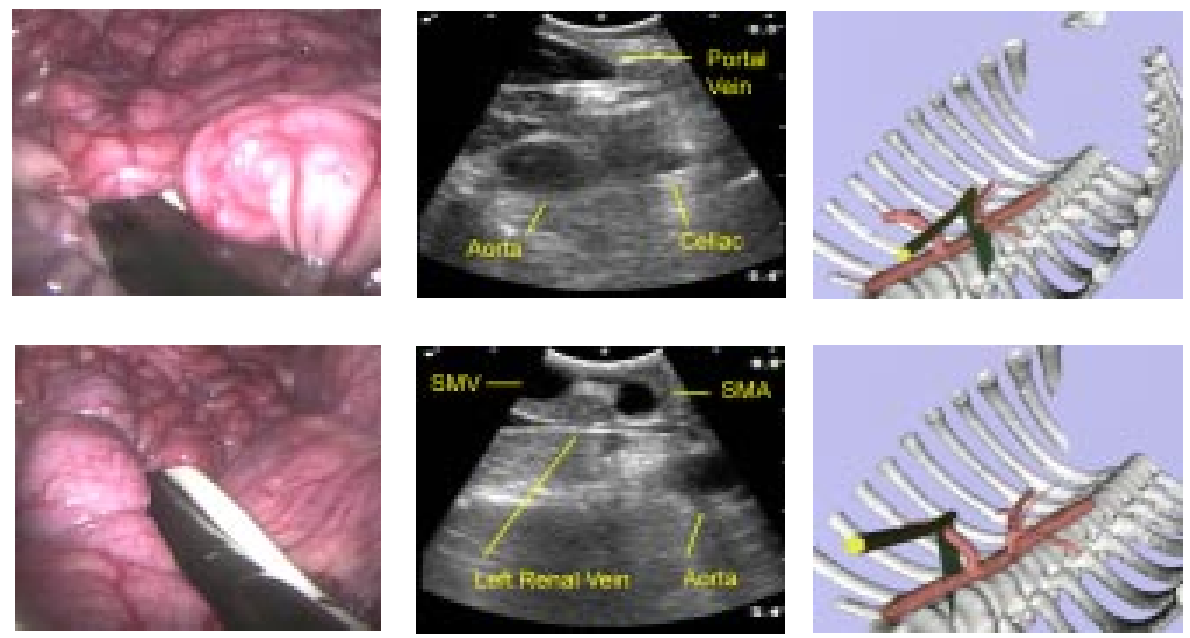

Fig. 1. The ultrasonographer is provided with three displays: the standard laparoscopic view (left), laparoscopic US (center), and real-time rendering of the ultrasound plane relative to the CT angiogram (right). The tip of the probe corresponds to the left side of the US image. The top row shows three still images from the video displays when the celiac axis is being imaged. The bottom row shows the superior mesenteric artery being imaged

\section{Navigation System}

The navigation system consists of four major hardware components: a laparoscopic transducer (Leopard; BK Medical, Wilmington, MA), a surgical pointer, a tracking device (miniBIRD; Ascension Technology, Burlington, VT), and a PIII-800Mhz laptop computer. Both the laparoscopic transducer and the surgical pointer are tracked using $5 \mathrm{~mm}$ electromagnetic sensorsADDIN. Figure 2 shows the tracking sensor mounted on the side of the laparoscopic transducer. Figure 3 shows how the system hardware is integrated. In the operating room, the transmitter for the tracking device is fixed to the side of the operating table approximately $50 \mathrm{~cm}$ from the imaged volume.

\section{Orientation Display}

The real time surgical visualization is a provided by custom module for 3D Slicer, an open source medical visualization package developed at MIT [9]. This module reads the data from the tracking device, computes the position and orientation of the US plane model, and renders the US plane model relative to models of the patient's aorta and ribs. The models of aorta and ribs were generated from the patient's preoperative contrast enhanced CT using segmentation tools in 3D Slicer. The models of the laparoscopic transducer and the B-Scan plane were generated using the Visualization Toolkit (Kitware, Clifton Park, NY). 


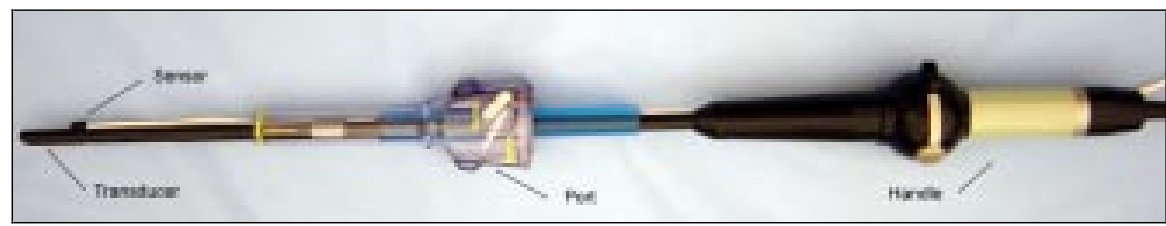

Fig. 2. The tip of the laparoscopic ultrasound probe is tracked using a $5 \mathrm{~mm}$ electromagnetic tracking sensor.

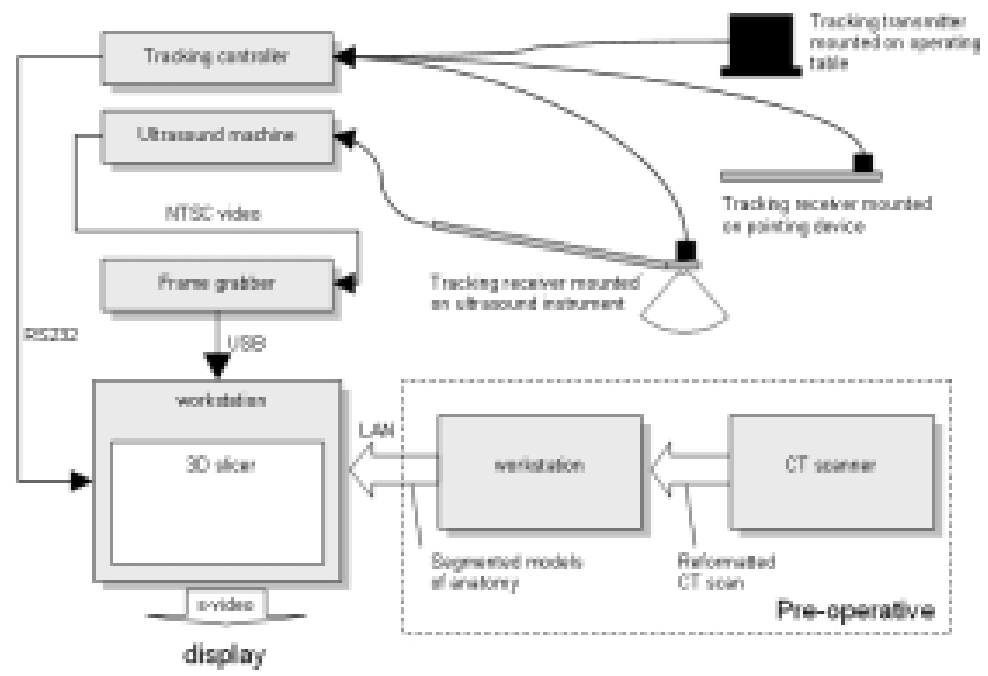

Fig. 3. A schematic of the system hardware components and data channels.

\section{Registration}

Registration was performed by computing a rigid transformation that mapped points on the patients in world coordinates into 3D Slicer coordinates. Four ribs tips were used as fiducials for the initial registration. We found that the tips of the $3^{\text {rd }}$ and $5^{\text {th }}$ lowest ribs gave the best registration. Structurally, these rib tips were more rigid compared to the lower ribs and easier to identify by palpation compared to the higher ribs. Their locations were recorded in world coordinates using the tracked pointer. ADDINTheir locations in 3D Slicer coordinates were obtained using a ray tracing technique for identifying points on models. The $4 \times 4$ matrix that defined transformation between the two point sets was computed using the Gauss-Newton algorithm.

Following initial registration, the ultrasonographer then proceeded with the laparoscopic examination. The system is designed so that the registration can be updated intraoperatively. The US was calibrated so it could be used to identify fiducials that could not be identified using the pointer. We routinely used the take off the celiac axis and superior mesenteric artery from the aorta to reduce position error. 
Both take off points are identified in the 3D Slicer coordinates and in the US coordinates. The mean Euclidean error is then added to the registration transformation. This technique was used to update the position registration but not the orientation. Due to the small size and close proximity of the arterial structures, we found minor errors in the update step could lead to large, inaccurate shifts in orientation. In effect, the ribs were used to provide gross position and orientation registration and the vessels are used to provide fine position registration. Figure 4 shows a flowchart which outlines the process we developed for visualization and registration.

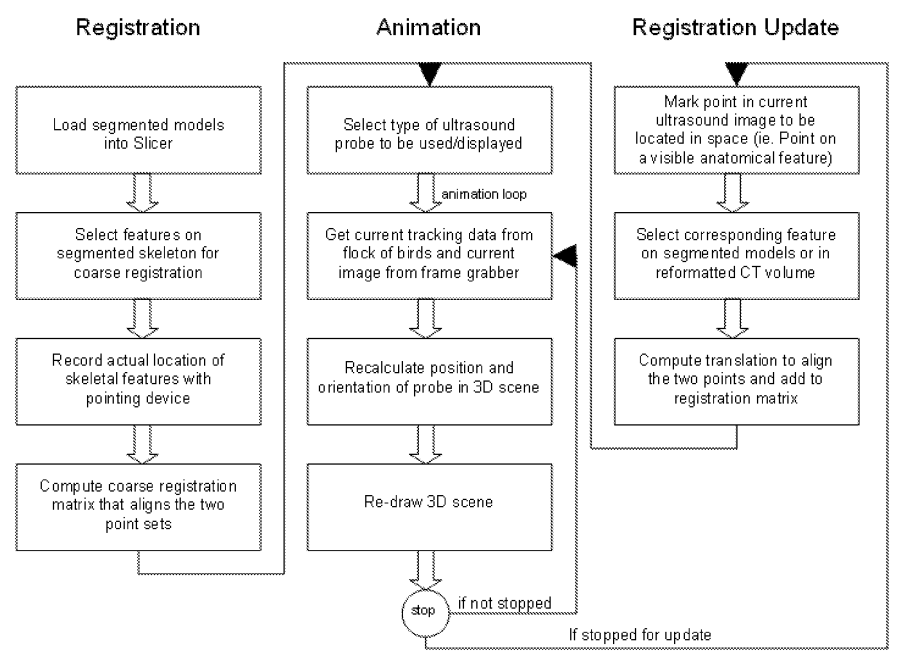

Fig. 4. A flowchart outlining the registration and visualization steps.

\section{Laparoscopic US Calibration}

We used the standard cross-wire technique to determine the calibration parameters, or the position $(x, y, z)$ and orientation $(\alpha, \beta, \gamma)$ of the ultrasound image plane with respect to the sensor [10]. With this approach, the intersection of two crossing wires in a water bath is used as a point phantom. The intersection is scanned while exercising all six degrees of freedoms of the tracking system. Let ${ }^{J} T_{I}$ be the transformation from any coordinate system $I$ to any coordinate system $J, P$ be the coordinate system of the B-scan plane, $R$ be the coordinate system of the position sensor, $T$ be the coordinate system of the transmitter, $u$ and $v$ be the column and row indices of the pixel in the cropped US image, $s_{x}$ and $s_{y}$ be scale factors. If the origin of a coordinate system $C$ is placed is placed at the intersection of the wires, the pixels $\left(P_{x}\right)$ at the center of the intersection should satisfy: $\left[\begin{array}{llll}0 & 0 & 0 & 1\end{array}\right]^{T}={ }^{C} T_{T}{ }^{T} T_{R}{ }^{R} T_{P} P_{x}$, where $P_{x}=\left[\begin{array}{llll}s_{x} u & S_{y} v & 0 & 1\end{array}\right]^{\mathrm{T}}$. We solved the resulting system of non-linear equations using the Gauss-Newton method. 


\section{Experiments}

To assess the performance of the tracking device when mounted on the side of laparoscopic transducer, 3D US volumes of a phantom were constructed and compared to a corresponding CT scan. The multi-modality phantom (CIRS, Norwalk, VA) used for the experiment is based on the upper abdominal anatomy. Two US volumes of the liver (left lobe, right lower lobe) were generated using Stradx, a 3D US application developed at Cambridge University [11]. The CT volume $(512 \times 512$, $1 \mathrm{~mm}$ slices) was obtained using a multi-detector row CT scanner (Somatoform Plus4; Siemens Medical Systems, Iselin, NJ). The registration of the 3D US and CT, and the analysis of the registration error was performed using 3D Slicer. Figure 5 shows an axial plane of registered 3D US and CT volumes. By comparing the position of distinct features within both volumes, we determined that the average registration error was less than $2 \mathrm{~mm}$ in the phantom.

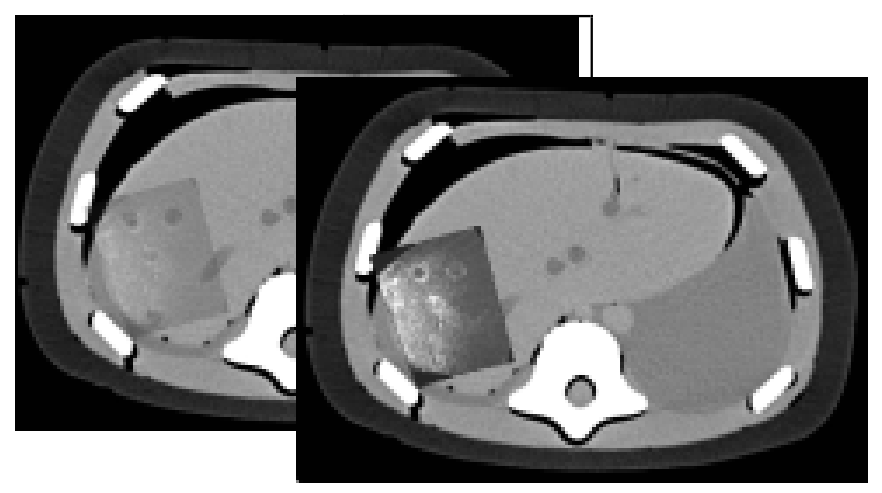

Fig. 5. A 3DUS of a phantom was generated and then registered with a corresponding CT scan to assess spatial accuracy

To assess the feasibility and utility of system, four physicians were asked to use the system while performing an exam on a $25 \mathrm{~kg}$ pig. The animal research protocol was approved by the Harvard Medical Area animal care committee. First a contrastenhanced CT was performed $[12,13]$. Immediately prior to the CT, the pig underwent endotracheal intubation and a general anesthetic. The pig was positioned in the CT scanner as it would be positioned on the operating table for a laparoscopy. During scanning, the pig was ventilated with oxygen and low tidal volume to minimize breathing motion artifact. The CT volume was obtained with a multi-detector row CT scanner (Somatoform Plus4). The helical scan extended from the xiphoid sternum to $2 \mathrm{~cm}$ below the lowest thoracic ribs. After a 20 gauge catheter was placed in the auricular vein, $50 \mathrm{ml}$ bolus of non-ionic contrast agent (Ultravist; Berlex laboratories, Wayne, NJ) was injected at $3 \mathrm{ml} / \mathrm{sec}$ using a power injector. The scanning parameters were: scan delay of $4 \mathrm{sec}, 120 \mathrm{kVp}, 52 \mathrm{~mA}, 1.25 \mathrm{~mm}$ collimation, table speed $30 \mathrm{~mm} / \mathrm{sec}$. The CT volume $(512 \times 512,2 \mathrm{~mm}$ slices $)$ was then transferred to the laptop 
and the arterial and skeletal anatomy was segmented using semi-automated approach prior to laparoscopic procedure.

The animal was brought to the operating room and following endotracheal intubation and a general anesthesic, it underwent laparoscopy and laparoscopic US. The animal and the CT volume were registered using the tip of the $3^{\text {rd }}$ and $5^{\text {th }}$ lowest ribs. A $10 \mathrm{~mm}$ access port for the laparoscopic camera was placed in the lower midline and the abdomen was insufflated to $15 \mathrm{mmHg}$ with $\mathrm{CO}_{2}$. A second $18 \mathrm{~mm}$ access port for the laparoscopic US was placed in left lower abdomen. The celiac axis and the superior mesenteric artery were identified in both US and CT images and the registration was updated. We assessed the registration error by comparing the position of arterial features in the 3D model to their actual locations in the animal seen on the ultrasound. Using the ribs as fiducials, we determined the average registration error was $5 \mathrm{~mm}$. The registration error was reduced to $3 \mathrm{~mm}$ when vasculature features were used to update the registration transformation.

Each physician then independently performed an exam. They were asked to identify four landmarks (celiac axis, superior mesenteric artery, portal vein confluence, and pancreatic duct) using standard laparoscopic feedback alone or in combination with the navigation system. The number of correct responses using standard laparoscopic feedback was compared to the correct responses using the navigation system with the Fisher's exact test. The physicians correctly identified more landmarks using the navigation system ( $69 \%$ vs. $25 \%, \mathrm{p}=0.02)$.

Off line testing was also performed to assess the utility of the navigation system. During a pig exam, video from the laparoscopic camera, laparoscopic US and navigation system was simultaneously digitized. From the recordings, twelve different synchronized clips of intra-abdominal anatomy were prepared. Two laparoscopic ultrasonographers reviewed the clips and established by consensus whether any of nine vessels were present. Twenty surgeons then independently reviewed the clips and were asked to identify whether any of the nine vessels were present. For all the clips, they were shown the laparoscopic view and the laparoscopic US. For half of the clips, they were also shown the orientation display. Whether the orientation display was shown for a particular clip was alternated for each successive surgeon. Accuracy was compared using the odds ratios (with $95 \%$ confidence intervals) from $2 \times 2$ tables. For all vessels, the orientation display improved the odds ratio for correctly identifying structures from $3.7(2.7$ - 5.2) to $8.9(6.2$ - 12.8). For arteries, the orientation display improved the odds ratio from $2.4(1.4-4.3)$ to $9.6(6.2-12.8)$. For veins, the orientation display improved the odds ratio from $4.4(2.8$ - 6.8) to 13.6 (7.225.7) There was no significant difference in the odds ratio if the tip of the probe was visible in the laparoscopic video clip. However, if the tip of the probe was not visible in the laparoscopic video clip (e.g., imaging the retroperitoneum), the orientation display improved the odds ratio from $2.3(1.4-3.8)$ to $12.4(7.2-21.4)$.

\section{Discussion}

We describe a novel system that tracks a laparoscopic transducer and displays the plane of the US image relative to a 3D model of the aorta. We also show that this system improves a physician's ability to correctly identify key structures in the US images. This system is helpful for surgeons learning to use laparoscopic US. It is also 
likely that this technique could help experienced laparoscopic ultrasonographers perform more challenging exams.

It is possible that the model of the aorta could be generated using transabdominal 3D US. The focus of this work though, was to assess the feasibility and utility of tracking a laparoscopic transducer and displaying the position and orientation of the US plane relative to a model of the aorta. Part of our ongoing work is assessing whether a model of the aorta generated from transabdominal 3D US could be an alternative approach for providing spatial cues that help physicians orient laparoscopic US images.

Acknowledgements. We thank Raul San Jose Estepar for his help configuring Stradx and Stuart Silverman for his helpful discussions on imaging. Funding for this research is provided by the U.S. Dept of the Army, under DAMD17-02-2-0006. We also acknowledge equipment support from BK Medical.

\section{References}

1. Birkett, D.H., Advanced display devices, in Cybersurgery : advanced technologies for surgical practice, R.M. Satava, Editor. 1998, Wiley-Liss: New York.

2. Harms, J., Feussner H, Baumgartner M, et al., Three-dimensional navigated laparoscopic ultrasonography. Surg Endosc, 2001. 15: p. 1459-1462.

3. Sato, Y., Miyamoto M, Nakamoto M, Nakajima Y, Shimada M, Hashizume M, Tamura S, 3D Ultrasound Image Acquisition Using Magneto-optic Hybrid Sensor for Laparoscopic Surgery. Lecture Notes in Computer Science, 2001(2208): p. 1151-1153.

4. Kane, R., Intraoperative, laparoscopic, and endoluminal ultrasound. 1999, Philadelphia: Churchill Livingstone. 224.

5. Gronningsaeter, A., et al., Initial experience with stereoscopic visualization of threedimensional ultrasound data in surgery. Surg Endosc, 2000. 14(11): p. 1074-8.

6. Ellsmere, J., Stoll JA, Rattner DW, Wells WM III, Kikinis R, Vosburg KG, Integrating preoperative CT data with laparosopic ultrasound images facilitates interpretation. Surg Endosc, 2003(17): p. S296.

7. Wagner, L.R., J. Ferwerda, and D. Greenberg, Perceiving Spatial Relationships in Computer-Generated Images. IEEE Comp. Graphics \& Applic, 1992: p. 44-58.

8. Sjolie, E., Kaspersen J, Weshe J, et al. Is minimally invasive abdominal surgery based on ultrasound vision possible? in Computer Assisted Radiology and Surgery. 2001.

9. 3D Slicer. 2002, www.slicer.org.

10. Prager, R.W., et al., Rapid calibration for 3-D freehand ultrasound. Ultrasound in Medicine and Biology, 1998. 24(6): p. 855-869.

11. Stradx. 2002, svr-www.eng.cam.ac.uk/ rwp/stradx/.

12. Dondelinger, R.F., et al., Relevant radiological anatomy of the pig as a training model in interventional radiology. Eur Radiol, 1998. 8(7): p. 1254-73.

13. Garcia, P., et al., Hepatic CT enhancement: effect of the rate and volume of contrast medium injection in an animal model. Abdom Imaging, 1999. 24(6): p. 597-603. 